\title{
Erratum to: MDMA-induced indifference to negative sounds is mediated by the $5-\mathrm{HT}_{2 \mathrm{~A}}$ receptor
}

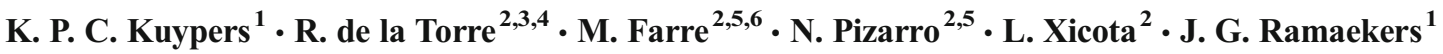

Published online: 1 August 2017

(C) Springer-Verlag GmbH Germany 2017

\section{Erratum to: Psychopharmacology}

DOI 10.1007/s00213-017-4699-1

In the original version of this article, one of the authors was missing its initial. Farre should be presented as M. Farre.

The original article was corrected

The online version of the original article can be found at http://dx.doi.org/10. 1007/s00213-017-4699-1

K. P. C. Kuypers

k.kuypers@maastrichtuniversity.nl

1 Department of Neuropsychology and Psychopharmacology, Faculty of Psychology and Neuroscience, Maastricht University, Maastricht, the Netherlands

2 Integrative Pharmacology and Neurosciences Systems Research Group, Institut Hospital del Mar d'Investigacions Mèdiques, Barcelona, Spain

3 Spanish Biomedical Research Centre in Physiopathology of Obesity and Nutrition (CIBEROBN), Santiago de Compostela, Spain

4 CEXS-UPF, Universitat Pompeu Fabra, Barcelona, Spain

5 Universitat Autonoma de Barcelona, Barcelona, Spain

6 Clinical Pharmacology, Hospital Universitari Germans Trias i Pujol, Badalona, Spain 